\title{
Artemisinin prevents electric remodeling following myocardial infarction possibly by upregulating the expression of connexin 43
}

\author{
YONGWEI GU ${ }^{1 *}$, GANG WU $^{2 *}, \mathrm{XIN} \mathrm{WANG}^{3}, \mathrm{XI} \mathrm{WANG}^{3}$, YULIN WANG $^{1}$ and CONGXIN HUANG ${ }^{2,3}$ \\ ${ }^{1}$ Department of Cardiology, The Fourth Affiliated Hospital of Nantong University (The First People's \\ Hospital of Yancheng), Yancheng, Jiangsu 224006; ${ }^{2}$ Department of Cardiology, Renmin Hospital of Wuhan \\ University; ${ }^{3}$ Cardiovascular Research Institute of Wuhan University, Wuhan, Hubei 430060, P.R. China
}

Received August 6, 2013; Accepted March 28, 2014

DOI: $10.3892 / \mathrm{mmr} .2014 .2431$

\begin{abstract}
Artemisinin has been demonstrated to exert beneficial effects on ventricular remodeling. The present study investigated whether artemisinin was able to decrease the ventricular fibrillation threshold (VFT) in rats following myocardial infarction (MI) and aimed to determine the possible underlying mechanisms. The rats were subjected to surgery to induce MI by ligation of the left anterior descending artery and were randomly allocated to receive vehicle or artemisinin $(75 \mathrm{mg} / \mathrm{kg} /$ day) treatment for four weeks. Programmed electrical stimulation demonstrated a significantly increased VFT in the artemisinin-treated group compared with the vehicle-treated group. The electrophysiological improvement of the VFT was accompanied by increased immunofluorescence-stained connexin 43 (Cx43), myocardial Cx43 protein and mRNA levels in artemisinin-treated rats. The present study also demonstrated that artemisinin significantly decreased tissue tumor necrosis factor (TNF)- $\alpha$ levels at the infarcted border zone. Thus, artemisinin demonstrated a protective effect on ventricular arrhythmias following MI. Although the precise mechanism by which artemisinin modulates the dephosphorylation of $\mathrm{Cx} 43$ is unknown, it is likely that artemisinin increased the expression of $\mathrm{Cx} 43$ via the inhibition of TNF- $\alpha$.
\end{abstract}

\section{Introduction}

It is well known that a lethal ventricular tachyarrhythmia, including ventricular fibrillation, is one of the most common reasons for sudden cardiac death following myocardial

Correspondence to: Professor Congxin Huang, Cardiovascular Research Institute of Wuhan University, 238 Jiefang Road, Wuhan, Hubei 430060, P.R. China

E-mail: huangcongxinwhu@163.com

*Contributed equally

Key words: artemisinin, myocardial infarction, connexin 43 infarction (MI). Multiple factors may contribute to the genesis of ventricular arrhythmia (VA); the electrical remodeling generates a proarrhythmogenic substrate following MI. The proarrhythmogenic substrate may be involved in altered ionic currents, action potentials, cardiac fibrosis and cell-to-cell coupling. Therefore, novel approaches that prevent electrical remodeling following $\mathrm{MI}$ are required.

Artemisinin is the active component of Artemisia annua L. and is approved worldwide for the treatment and prevention of malaria (1). In addition to its antimalarial properties, previous studies have demonstrated that artemisinin significantly inhibits ventricular remodeling. The present study demonstrated that artemisinin attenuates ventricular remodeling and neural remodeling following MI by exhibiting anti-inflammatory effects $(2,3)$. However, whether artemisinin is able to attenuate electrical remodeling following MI remains unclear. In order to assess our hypothesis, a rat MI model was used to determine whether artemisinin prevents electrical remodeling following MI.

\section{Materials and methods}

Animal preparation. All experiments were approved by the Institutional Animal Care and Use Committee of Wuhan University (Wuhan, China) and were conducted in accordance with the Guideline for the Care and Use of Laboratory Animals.

Adult male Sprague-Dawley rats (Center for Animal Experiments, Wuhan University, Wuhan, China) weighing 250-300 g were anesthetized with an intraperitoneal injection of $3 \%$ pentobarbital sodium $(30 \mathrm{mg} / \mathrm{kg})$. A left intercostal thoracotomy was performed to expose the heart and the left anterior descending (LAD) artery was ligated at the origin. The sham group underwent thoracotomy and pericardiotomy; however, not LAD ligation (4). The chest was then closed and the animals were allowed to recover in a warm, clean cage. Fatalities within $24 \mathrm{~h}$ of surgery were excluded from the present study.

Reagents. Artemisinin was purchased from the Guilin Pharmaceutical Factory (Guangxi, China). The drug was dissolved in $0.5 \%$ carboxymethyl cellulose (CMC) immediately prior to use. The drug safety of artemisinin up to 28 days 
was presented in a previous study (5). The vehicle $(0.5 \% \mathrm{CMC})$ was used as a control.

Treatment protocol. The rats surviving $24 \mathrm{~h}$ following MI were randomly assigned to two treatment groups as follows: $75 \mathrm{mg} / \mathrm{kg} /$ day artemisinin by oral gavage three times a day for four weeks (MI + artemisinin group; $n=21)$; the same volume of $0.5 \% \mathrm{CMC}$ liquid vehicle by oral gavage three times a day for four weeks after MI (MI + vehicle group; $\mathrm{n}=21$ ) and the same treatment as the MI + vehicle group in the sham operation group $24 \mathrm{~h}$ after sham operation (thoracotomy with LAD isolation, however, without ligation; $\mathrm{n}=10$ ) for four weeks.

Measurement of tumor necrosis factor (TNF)- $\alpha$ levels. Blood $(0.75 \mathrm{ml})$ was collected into chilled EDTA tubes one day prior to MI surgery and subsequently on days $1,3,5,7,14$, 21 and 28 after surgery. The blood samples were centrifuged at $1,100 \times \mathrm{g}, 4^{\circ} \mathrm{C}$ and the plasma samples were separated and stored at $-70^{\circ} \mathrm{C}$ until they were assayed for TNF- $\alpha$. Red blood cells were suspended in an equal volume of heparinized saline and reinfused.

The TNF- $\alpha$ levels in the rats were measured using an ultrasensitive rat TNF- $\alpha$ ELISA kit (Biosource International, Inc., Camarillo, CA, USA) according to the manufacturer's instructions. The details of the methodology were described in a previous study (6).

Electrophysiological evaluation. To assess the inducibility of VAs, the burst stimuli was used $(2 \mathrm{~ms}$ pulses at $50 \mathrm{~Hz}, 2 \mathrm{sec}$ burst duration) and burst pacing was used for up to $3 \mathrm{~min}$ of pacing in the infarcted border zone (IBZ).

To assess the ventricular fibrillation threshold (VFT), electrical stimulation was supplied with $100 \mathrm{~Hz}$ to the right ventricle. Each stimulation was administered for $30 \mathrm{sec}$. The interval between each episode of stimulation was $1 \mathrm{~min}$. The initial pacing voltage was $1 \mathrm{~V}$ and progressively increased by $0.5 \mathrm{~V}$. VFT was defined as the lowest voltage at which ventricular fibrillation was induced and sustained for at least $20 \sec (7)$.

Quantitative polymerase chain reaction ( $q P C R$ ). Total RNA was extracted from the non-infarcted zone (NIZ) and the IBZ of the left ventricle using TRIzol reagent (Invitrogen Life Technologies, Carlsbad, CA, USA) according to the manufacturer's instructions. qPCR was performed using the ABI Prism 7000 (Applied Biosystems, Foster City, CA, USA). cDNA was amplified under the following conditions: $94^{\circ} \mathrm{C}$ for $10 \mathrm{~min}$ and then for 45 cycles at $94^{\circ} \mathrm{C}$ for $10 \mathrm{sec}$ and $56^{\circ} \mathrm{C}$ for $30 \mathrm{sec}$. The primer sequences of connexin $43(\mathrm{Cx} 43)$ and glyceraldehyde-3-phosphate dehydrogenase (GAPDH) are shown in Table I. For quantification, Cx43 expression was normalized to GAPDH. The reactions were programmed on a computer linked to the detector (ABI Prism 7000 Sequence Detection system; Applied Biosystems) for 40 cycles of the amplification step. Experiments were replicated three times and the results are expressed as the mean value.

Western blot analysis of Cx43. The peri-infarcted zone of the left ventricle and the same zone of the left ventricle in the sham
Table I. Primer sequences for Cx43 and actin.

\begin{tabular}{lc}
\hline Gene & \multicolumn{1}{c}{ Primer sequence } \\
\hline Cx43 & \\
Forward & ACAGCGCAGAGCAAAATCG \\
Reverse & ATGGCTGGAGTTCATGTCCAG \\
Actin & \\
Forward & GCTCCTCCTG AGCGCAAGTA \\
Reverse & CCTGCTTGCTGATCCACATCT \\
\hline
\end{tabular}

Cx43, connexin 43 .

group were used for western blot analysis. Equal quantities of protein were loaded and separated by SDS-PAGE, transferred onto a nitrocellulose membrane and incubated with primary antibodies (anti-Cx43, polyclonal antibody; dilution 1:1,000; Abcam, Cambridge, MA, USA) and anti-GAPDH (1:2,000; Abcam) overnight at $4^{\circ} \mathrm{C}$. Horseradish peroxidase-conjugated anti-rabbit immunoglobulin $\operatorname{IgG}(1: 1,500$; Beyotime Institute of Biotechnology, Shanghai, China) was applied as the secondary antibody for $1 \mathrm{~h}$ at room temperature. Finally, the blots were visualized using an enhanced chemiluminescence kit (Beyotime Institute of Biotechnology) and the signals were analyzed using a Bio-Rad image system (Bio-Rad, Hercules, CA, USA).

Immunofluorescent studies of Cx43. Immunofluorescence staining was performed to investigate the localization and the distribution of $\mathrm{Cx} 43$ at the peri-infarct zone of the left ventricle. Fresh tissue was fixed in $4 \%$ paraformaldehyde for $24 \mathrm{~h}$, then dehydrated in $30 \%$ sucrose for $48 \mathrm{~h}$. The tissue was then embedded at $-25^{\circ} \mathrm{C}$ (frozen section inside the machine) and then frozen sectioning was performed. Following inhibition with goat serum for $30 \mathrm{~min}$, the frozen slices ( $5 \mu \mathrm{m}$ thick) were incubated with rabbit polyclonal anti-Cx43 antibody (Zymed Life Technologies, Carlsbad, CA, USA) overnight at $4^{\circ} \mathrm{C}$ and then incubated with fluorescein isothiocyanate (FITC)-conjugated anti-rabbit IgG (Zymed Life Technologies) for $1 \mathrm{~h}$ at room temperature. The specimens were examined under a Leica M205 FA fluorescence microscope (Leica, Wetzlar, Germany).

Statistical analysis. All values are expressed as the mean \pm standard deviation. Comparisons between groups were performed using one way analysis of variance and the least significant difference test was used for post hoc multiple comparisons. $\mathrm{P}<0.05$ was considered to indicate a statistically significant difference.

\section{Results}

Animal survival rate and infarct size. At 28 days after MI, the survival rate in the artemisinin treatment group (68.88\%; $31 / 45$ ) was significantly higher than that in the vehicle-treated group $(42.22 \%, 19 / 45 ; \mathrm{P}<0.05)$. The infarct size four weeks after MI was similar between the $\mathrm{MI}+$ artemisinin group and the MI + vehicle group $(34.32 \pm 1.68 \%$ and $33.18 \pm 1.42 \%$, respectively; $\mathrm{P}>0.05)$. 


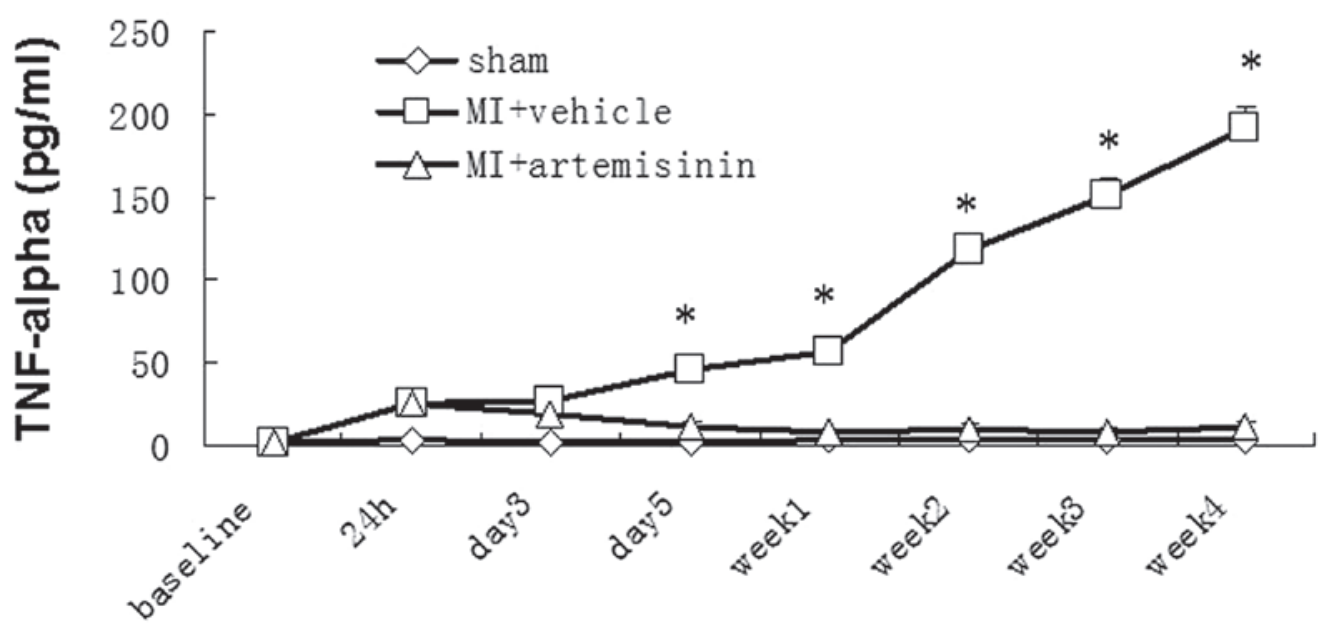

Figure 1. Plasma TNF- $\alpha$ levels among three groups following MI in rats with vehicle or artemisinin treatment for 4 weeks. TNF- $\alpha$ levels were comparable among the three groups at baseline. In the vehicle-treated group, TNF- $\alpha$ levels increased within $24 \mathrm{~h}$ after MI and remained elevated throughout the study. In the artemisinin treatment group, threre was a similar early increase in TNF- $\alpha$ within 24 h; however, TNF- $\alpha$ was reduced to baseline levels by day 4 of artemisinin treatment and remained low throughout the rest of the study. In the sham-operation group, the TNF- $\alpha$ levels remained at or around baseline throught the study. ${ }^{~} \mathrm{P}<0.05$ vs. the sham group and the artemisinin treatment group. TNF- $\alpha$, tumor necrosis factor- $\alpha$; MI, myocardial infarction.

Plasma TNF- $\alpha$ levels. As shown in Fig. 1, the baseline plasma TNF- $\alpha$ level $(\mathrm{pg} / \mathrm{ml})$ in the three groups observed were comparable across groups $(\mathrm{P}>0.05)$. In the sham group, TNF- $\alpha$ levels remained at or around baseline during the entire study. By contrast, in the $\mathrm{MI}+$ vehicle group the TNF- $\alpha$ level was increased within $24 \mathrm{~h}$ after MI (25.6 \pm 4.2$)$ and remained elevated $(26.71 \pm 4.11,46.18 \pm 4.7,56.42 \pm 3.47$, $117.52 \pm 8.72,150.8 \pm 11.31,191.08 \pm 12.68$ on days $3,5,7$, 14, 21, 28 after MI, respectively) throughout the study. The TNF- $\alpha$ level in the MI + artemisinin group also increased within $24 \mathrm{~h}(24.46 \pm 6.15)$, however, decreased (18.24 \pm 3.24$)$ on day 3 , returned to baseline by day 5 and then remained low $(10.31 \pm 3.09,7.72 \pm 2.08,9.95 \pm 2.37,8.08 \pm 2.49,10.33 \pm 3.08)$ throughout the study.

Inducibility of VAs and VFT. The VA was induced by burst stimuli, compared with the sham group, the inducibility was significantly higher in the MI + vehicle group; however, the inducibility was reduced in the artemisinin treatment group $(\mathrm{P}<0.05)$. VFT, reflects the vulnerability of ventricular fibrillation and VFT was significantly decreased in the MI + vehicle group and only marginally decreased in the $\mathrm{MI}+$ artemisinin group (Fig. 2).

qPCR of Cx43. The Cx43 mRNA levels 4 weeks after MI demonstrated a significant downregulation at the IBZ in the vehicle-treated group compared with the sham group. However, the reduction of Cx43 mRNA expression in the artemisinin-treated group following MI was significantly smaller than the reduction observed in the vehicle-treated group (Fig. 3).

Western blot analysis. The level of Cx43 protein was investigated by western blotting. Representative blots and quantitative results are presented in which $\mathrm{Cx} 43$ band intensities are normalized to $\beta$-actin. Two predominant forms of $\mathrm{Cx} 43$ were detected, the phosphorylated form of $\mathrm{Cx} 43$ (P-Cx43;
$43 \mathrm{kDa}$ ) and the non-phosphorylated form of Cx43 (NP-Cx43; $41 \mathrm{kDa})$. Compared with the sham group, the $\mathrm{P}-\mathrm{Cx} 43 / \beta$-actin was significantly reduced in the $\mathrm{MI}+$ vehicle group. By contrast, the decrease in $\mathrm{P}-\mathrm{Cx} 43 / \beta$-actin was reversed in the $\mathrm{MI}+$ artemisinin group (Fig. 4).

Immunofluorescent studies of Cx43. The connexin localization was evaluated using an immunofluorescent technique. Cx43 protein was only located at the intercalated disk area in the left ventricle of the sham group or normal tissue far from the infarcted zone. However, the distribution of $\mathrm{Cx} 43$ on the IBZ was disrupted. In the areas of the IBZ, Cx43 was distributed on the lateral side of the myocyte, however, not in the intercalated disk area. The disarray of Cx43 was ameliorated in the IBZ of the artemisinin treatment group, where its distribution was relatively normal. Quantitative analysis of the Cx43-signal intensity demonstrated that the Cx43-signal in the vehicle-treated group was markedly reduced in the IBZ, however, was partially attenuated by artemisinin treatment (Fig. 5).

\section{Discussion}

To the best of our knowledge, the present study demonstrated for the first time that artemisinin is able to ameliorate electrical remodeling at the IBZ following MI. Firstly, artemisinin treatment significantly inhibited the reduction of total Cx43 and phosphorylated Cx43. Secondly, the ischemia-induced disarrangement and distribution were reversed following artemisinin treatment. Thirdly, artemisinin treatment reduced the inducibility of VAs and increased VFT in rats with MI.

Artemisinin is the active component of Artemisia annua L. and is approved worldwide for the treatment and prevention of malaria (1). Previous studies have indicated that artemisinin is important in cardioprotection, and that it is able to attenuate ventricular remodeling and neural 

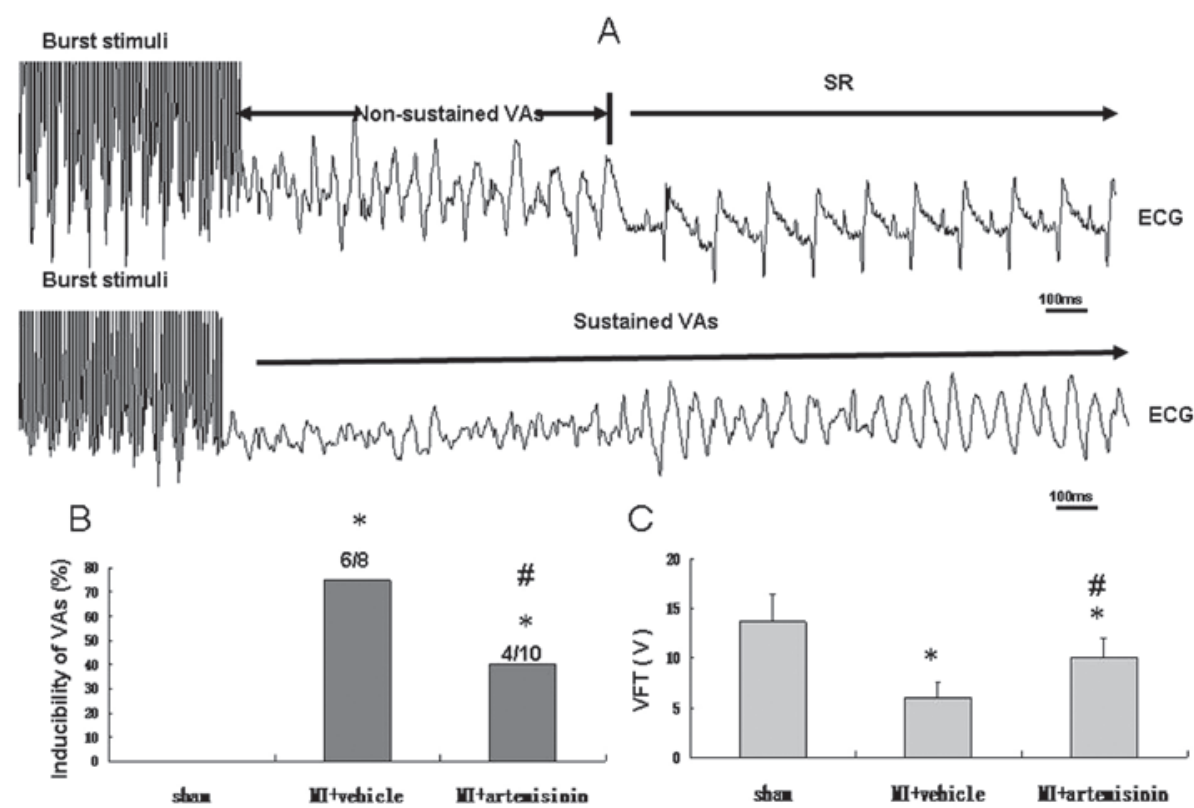

Figure 2. Inducibility of VAs by burst stimulation and the VFT by burst stimulation with increased pacing voltage. (A) Representative sustained ventricular arrhythmias induced by ventricular pacing. (B) The inducibility of ventricular arrhythmias (VAs) was significantly lower in the artemisinin-treated group than the vehicle-treated group. (C) VFT was reduced in the MI + vehicle group and marginally reduced in the MI + artemisinin group. VFT, ventricular fibrillation threshold; VAs, ventricular arrhythmias.

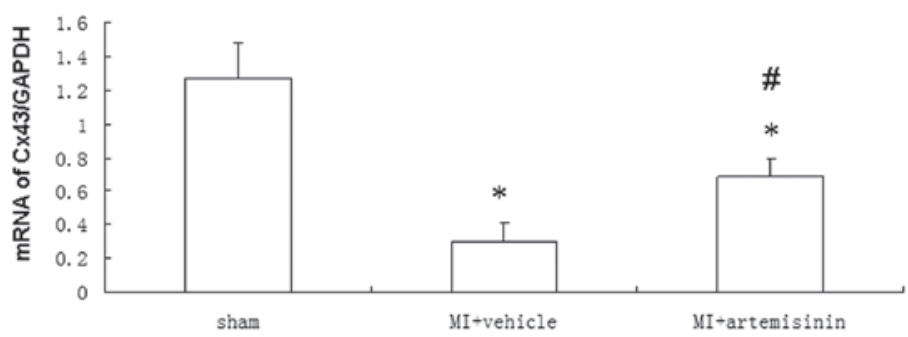

Figure 3. qPCR analysis of $\mathrm{Cx} 43$ following MI. Each mRNA was normalized to the mRNA level of GAPDH. Each column and bar represent the mean \pm standard deviation. ${ }^{*} \mathrm{P}<0.05$, compared with the sham group, ${ }^{\#} \mathrm{P}<0.05$, compared with the $\mathrm{MI}+$ vehicle group. Cx43, connexin 43 ; MI, myocardial infarction; GAPDH, glyceraldehyde-3-phosphate dehydrogenase; qPCR, quantitative polymerase chain reaction.

A
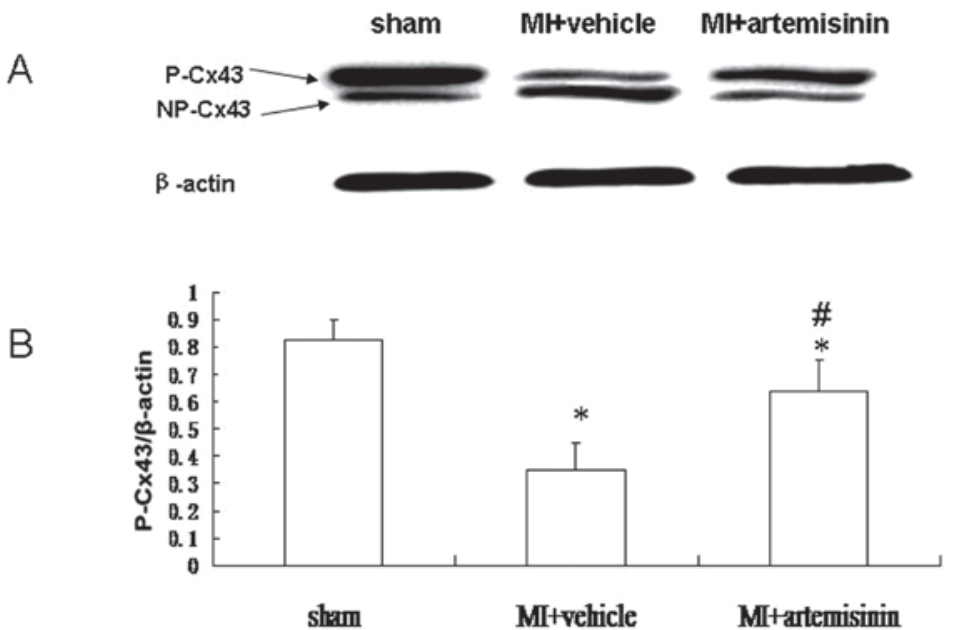

Figure 4. Western blot analysis of Cx43 protein from the infarcted border zone of the left ventricle 4 weeks after MI. (A) Representative immunoblots from the sham group, the MI + vehicle group and the MI + artemisinin group, probed with polyclonal $\mathrm{Cx} 43$ antibody. Arrows indicate the position of the phosphorylated isoform of Cx43 (43 kDa) and the nonphosphorylated isoform of Cx43 (41 kDa) bands, respectively. (B) Quantitative densitometric analysis of the phosphorylated isoform of $\mathrm{Cx} 43$. Data are expressed as the mean \pm standard deviation. ${ }^{*} \mathrm{P}<0.05$, compared with the sham group, ${ }^{\#} \mathrm{P}<0.05$, compared with the MI + vehicle group. Cx43, connexin 43; MI, myocardial infarction. 

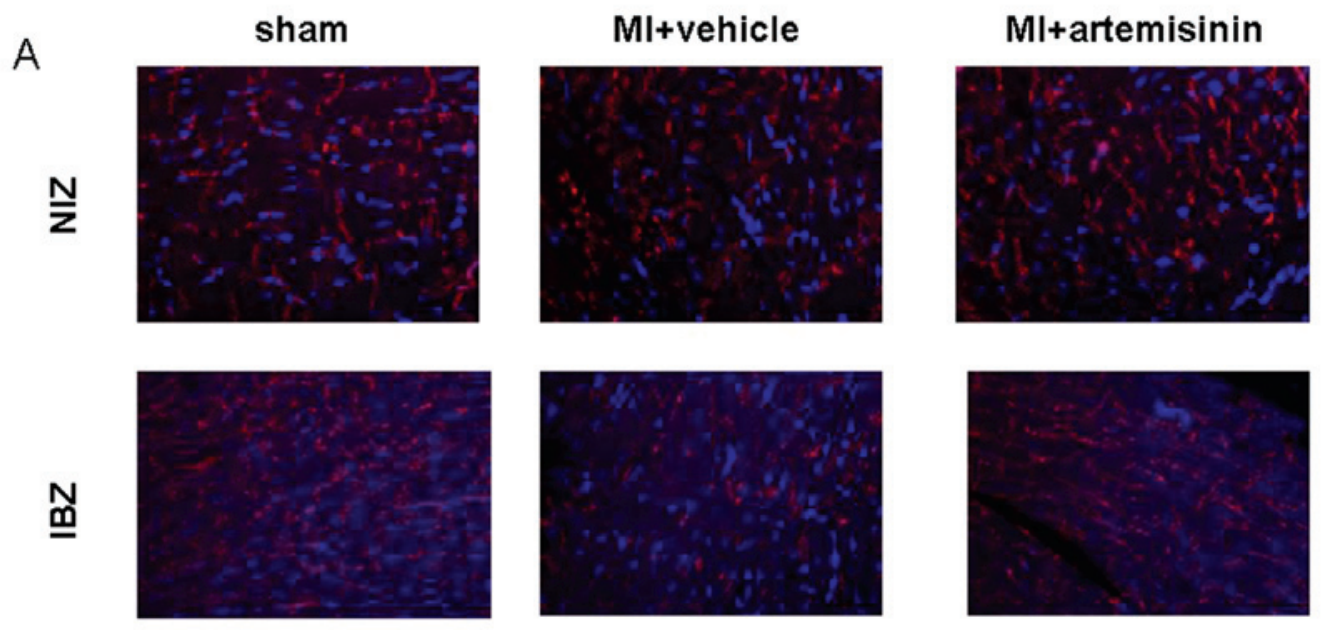

B

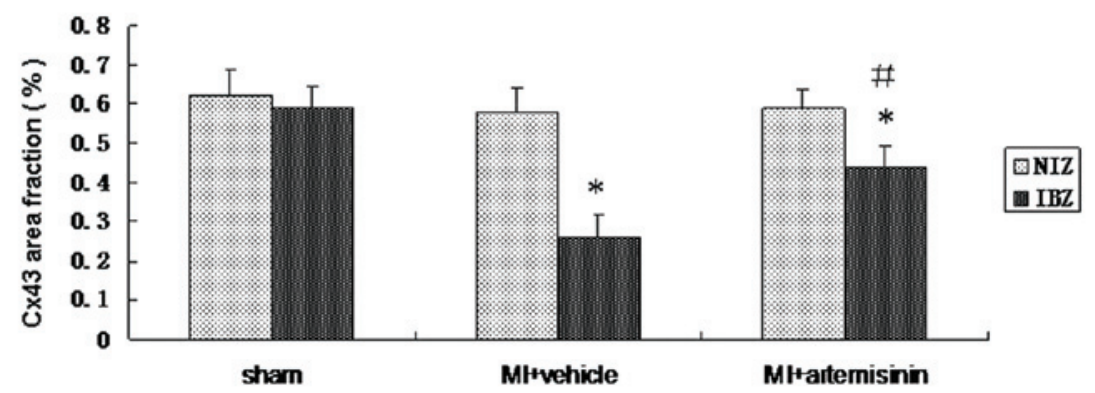

Figure 5. Immunofluorescent studies of Cx43 following MI. (A) Cx43 signals were mainly located at end-to-end apposition among the neighboring cells in the sham group. By contrast, MI markedly decreased the expression of Cx43 in the IBZ; however, not in the NIZ. Following administration of artemisinin, Cx43 signals were increased in the IBZ. Blue fluorescence indicates nuclei (DAPI) and red indicates Cx43 (rabbit polyclonal anti-Cx43 antibody). (B) Proportion of the total cell area occupied by $\mathrm{Cx} 43$ immunoreactive signals at the border zone. Data are expressed as the mean \pm standard deviation. ${ }^{*} \mathrm{P}<0.05$, compared with the sham group, ${ }^{\#} \mathrm{P}<0.05$, compared with the $\mathrm{MI}+$ vehicle group. Cx43, connexin 43; MI, myocardial infarction; IBZ, infarcted border zone; NIZ, non-infarcted zone.

remodeling (2-3). The present study demonstrated the antiarrhythmic effect of artemisinin.

It is well known that $\mathrm{Cx} 43$, which is the predominant ventricular gap junction protein, is critical for maintaining normal cardiac electrical conduction. Cardiac-restricted knockout of $\mathrm{Cx} 43$ causes a slowed ventricular conductive velocity and spontaneous VAs (8-9). Apart from reducing conduction, Cx43 remodeling may be associated with action potential duration dispersion in the failing heart (10). Furthermore, increased $\mathrm{Cx} 43$ by either $\mathrm{Cx} 43$ gene transfer or transplantation of embryonic cardiomyocytes decreases the spatial heterogeneity of repolarization and has the potential to reduce life-threatening post-infarct arrhythmias (11-12). Post-MI, the epicardial border zone (EBZ) demonstrated a marked disruption in gap-junctional distribution, with $\mathrm{Cx} 43$ disarrayed along the lateral surfaces of the cells (13) and selectively reduced in the EBZ (14). The present study observed that the disarray of $\mathrm{Cx} 43$ was ameliorated in the IBZ of the artemisinin-treated heart, displaying a relatively normal distribution. In addition, the present study demonstrated that the Cx43 mRNA level and the quantity of $\mathrm{Cx} 43$ protein were significantly increased at the IBZ by artemisinin therapy. The beneficial effects on $\mathrm{Cx} 43$ may improve electrical conduction and ameliorate repolarization dispersion in the IBZ. It may provide a rational explanation for why artemisinin prevented the induction of VAs in the present study.
Although the present study suggests that the mechanisms by which artemisinin produces anti-arrhythmic effects are associated with increases in $\mathrm{Cx} 43$ expression, other potential mechanisms require investigation. Firstly, our previous studies demonstrated that artemisinin attenuates interstitial fibrosis (2) and sympathetic reinnervation in the IBZ (3), which contributes to VAs and sudden cardiac death. Secondly, previous studies have confirmed that artemisinin exerts a direct effect on the ion channels (15-16). Therefore, it was hypothesized that artemisinin produces anti-arrhythmic effects by directly regulating the cardiac ionic channel.

Several potential mechanisms may be involved in the ability of artemisinin to inhibit Cx43 degradation and increase Cx43 expression following MI. It has been demonstrated that myocardial ischemia with sympathetic nerve stimulation promoted the degradation of the Cx43 protein (17) and vagal nerve stimulation protects the heart against ischemic-induced arrhythmias by preserving $\mathrm{Cx} 43$ protein (18), suggesting that autonomic nerve activity correlates with the survival time of $\mathrm{Cx} 43$. Our previous study revealed that artemisinin inhibits neural remodeling and sympathetic hyperinnervation following MI in rats (3). One mechanism by which artemisinin inhibits the degradation of Cx43 following MI may be by directly attenuating sympathetic tone. Secondly, artemisinin is able to upregulate the mRNA level of $\mathrm{Cx} 43$, indicating that increasing $\mathrm{Cx} 43$ protein at the transcriptional 
level may serve as another mechanism. Thirdly, inflammatory factors, including TNF- $\alpha$, which is able to aggravate the dephosphorylation and degradation of $\mathrm{Cx} 43$ (19), were increased significantly following MI. Artemisinin is able to inhibit the levels of TNF- $\alpha$ following MI, which contributes to the upregulation of the $\mathrm{Cx} 43$ protein.

There are several limitations in the present study. Firstly, the transmural heterogeneity of $\mathrm{Cx} 43$ expression was not evaluated in the present study, such an analysis is required to clarify the precise mechanisms for the anti-arrhythmic effects of artemisinin. Secondly, although artemisinin was demonstrated to attenuate the reduction of $\mathrm{Cx} 43$ possibly by reducing TNF- $\alpha$ levels in the IBZ, the present study did not provide direct evidence by evaluating the effect of artemisinin on $\mathrm{Cx} 43$ expression following inhibition of TNF- $\alpha$ expression. Finally, the cardiac morphology and hemodynamics were not measured at the end of the study, although the results were demonstrated in our previous study (2).

In conclusion, artemisinin reduces the vulnerability to VAs and increases the VFT following MI. The cardioprotective effects of artemisinin may occur by reducing TNF- $\alpha$ levels and preventing gap junction remodeling. The results suggest that artemisinin is a potential therapeutic candidate for the prevention of ventricular electrical remodeling following MI.

\section{Acknowledgements}

This study was supported by the Fundamental Research funds for the Central Universities (grant no. 201130202020003), the Natural Science Foundation of Hubei province, China (grant no. 2011CHB034), the National Natural Science Foundation of China (grant no. 81270305) and the National Science \& Technology Pillar Program of China (grant no. 2011BAI11B12).

\section{References}

1. Haynes, RK: Artemisinin and derivatives: the future for malaria treatment? Curr Opin Infect Dis 14: 719-726, 2001.

2. Gu Y, Wang X, Wang X, Yuan M, Wu G, Hu J, Tang Y and Huang C: Artemisinin attenuates post-infarct myocardial remodeling by down-regulating the NF- $\kappa \mathrm{B}$ pathway. Tohoku J Exp Med 227: 161-170, 2012.

3. Gu Y, Wang X, Wu G, Wang X, Cao H, Tang Y and Huang C: Artemisinin suppresses sympathetic hyperinnervation following myocardial infarction via anti-inflammatory effects. J Mol Histol 43: 737-743, 2012

4. Wen HZ, Jiang H,Li L, Xie P,Li JY,Lu Zb and He B: Semaphorin 3A attenuates electrical remodeling at infarct border zones in rats after myocardial infarction. Tohoku J Exp Med 225: 51-57, 2011.
5. Xiong Z, Sun G, Zhu C, Cheng B, Zhang C, Ma Y and Dong Y: Artemisinin, an anti-malarial agent, inhibits rat cardiac hypertrophy via inhibition of NF- $\kappa \mathrm{B}$ signaling. Eur J Pharmacol 649: 277-284, 2010.

6. Ponnappa BC, Dey I, Tu GC, Zhou F, Aini M, Cao QN and Israel Y: In vivo delivery of antisense oligonucleotides in $\mathrm{pH}$-sensitive liposomes inhibits lipopolysaccharide-induced production of tumor necrosis factor- $\alpha$ in rats. J Pharmacol Exp Ther 297: 1129-1136, 2001.

7. Wen H, Jiang H, Lu Z, He B, Hu X, Chen J and Zhao D: Carvedilol ameliorates the decreases in connexin 43 and ventricular fibrillation threshold in rats with myocardial infarction. Tohoku J Exp Med 218: 121-127, 2009.

8. Lerner DL, Yamada KA, Schuessler RB and Saffitz JE: Accelerated onset and increased incidence of ventricular arrhythmias induced by ischemia in $\mathrm{Cx} 43$-deficient mice. Circulation 101: 547-552, 2000.

9. Yao JA, Gutstein DE, Liu F, Fishman GI and Wit AL: Cell coupling between ventricular myocyte pairs from connexin43-deficient murine hearts. Circ Res 93: 736-743, 2003.

10. Poelzing S and Rosenbaum DS: Altered connexin43 expression produces arrhythmia substrate in heart failure. Am J Physiol Heart Circ Physiol 287: H1762-H1770, 2004.

11. Amino M, Yoshioka K, Tanabe T, Tanaka E, Mori H, Furusawa Y, et al: Heavy ion radiation up-regulates $\mathrm{Cx} 43$ and ameliorates arrhythmogenic substrates in hearts after myocardial infarction. Cardiovasc Res 72: 412-421, 2006.

12. Fernandes S, van Rijen HV, Forest V, Evain S, Leblond AL, Mérot J, et al: Cardiac cell therapy: overexpression of connexin 43 in skeletal myoblasts and prevention of ventricular arrhythmias. J Cell and Mol Med 13: 3703-3712, 2009.

13. Peters NS, Coromilas J, Severs NJ and Wit AL: Disturbed connexin43 gap junction distribution correlates with the location of reentrant circuits in the epicardial border zone of healing canine infarcts that cause ventricular tachycardia. Circulation 95: 988-996, 1997.

14. Ohara T, Ohara K, Cao JM, et al: Increased wave break during ventricular fibrillation in the epicardial border zone of hearts with healed myocardial infarction. Circulation 103: 1465-1472, 2001.

15. Qiao G, Li S, Yang B and Li B: Inhibitory effects of artemisinin on voltage-gated ion channels in intact nodose ganglion neurones of adult rats. Basic Clin Pharmacol Toxicol 100: 217-224, 2007.

16. Yang BF, Luo DL, Bao LH, Zhang YC and Wang HZ: Artemisinin blocks activating and slowly activating $\mathrm{K}^{+}$current in guinea pig ventricular myocytes. Zhongguo Yao Li Xue Bao 19: 269-272, 1998.

17. Jiang H, Hu X, Lu Z, Wen H, Zhao D, Tang Q, et al: Effects of sympathetic nerve stimulation on ischemia-induced ventricular arrhythmias by modulating connexin 43 in rats. Arch Med Res 39: 647-654, 2008.

18. Ando M, Katare RG, Kakinuma Y, Zhang D, Yamasaki F, Muramoto $\mathrm{K}$, et al: Efferent vagal nerve stimulation protects heart against ischemia-induced arrhythmias by preserving connexin43 protein. Circulation 112: 164-170, 2005.

19. Hao JL, Suzuki K, Lu Y, Hirano S, Fukuda K, Kumagai N, Kimura $\mathrm{K}$ and Nishida T: Inhibition of gap junction-mediated intercellular communication by TNF-alpha in cultured human corneal fibroblasts. Invest Ophthalmol Vis Sci 46: 1195-1200, 2005. 